\title{
Segmentation of Cerebrospinal Fluid from 3D CT Brain Scans Using Modified Fuzzy C-Means Based on Super-Voxels
}

\author{
Abdelkhalek Bakkari \\ Lodz University of Technology \\ Insitute of Applied Computer Science \\ 18/22 Stefanowskiego Str., \\ 90-924 Lodz, Poland \\ Email: bakkari.abdelkhalek@hotmail.fr
}

\author{
Anna Fabijańska \\ Lodz University of Technology \\ Insitute of Applied Computer Science \\ 18/22 Stefanowskiego Str., \\ 90-924 Lodz, Poland \\ Email: anna.fabijanska@p.lodz.pl
}

\begin{abstract}
In this paper, the problem of segmentation of 3D Computed Tomography (CT) brain datasets is addressed using the fuzzy logic rules. In particular, a new method which combines Fuzzy C-Means clustering and the idea of super-voxels is introduced. Firstly, the method applies the extended Simple Linear Iterative Clustering (SLIC) method to divide image into supervoxels, which are next clustered by Modified Fuzzy C-Means algorithm. The method deals with 3D images and performs fully three dimensional image segmentation. Ten samples are supplied proving that our Modified Fuzzy C-Means (MFCM) together with super-voxels are apt to take into account a large diversity of special domains that appear and which are inappropriate solved adopting classical Fuzzy C-Means approach. The results of applying the introduced method to segmentation of the CerebroSpinal Fluid (CSF) from the brain ventricles are presented and discussed.
\end{abstract}

\section{INTRODUCTION}

D IVIDING an image into coherent regions, that are somehow homogeneous and uniform leads to image segmentation.

One of the most popular clustering algorithms used for image segmentation is the Fuzzy C-Means (FCM) approach [1]. Since the method has a lot of advantages (e.g. it provides the best results for overlapped data sets of pixels) it is especially popular when the segmentation of medical images is required [2]. In particular, there was a significant number of attempts to apply FCM clustering for brain segmentation [3], [4], [5]. These works however consider mainly MRI datasets. To the best of our knowledge, there are only few works regarding brain segmentation from $\mathrm{CT}$ datasets.

Despite its popularity, the FCM algorithm has also some disadvantages, which limit its application to segmentation of 3D CT medical datasets. The main limitation of the algorithm is in particular its high computational complexity, intensive memory workload and unacceptably long time of computations. These result from the necessity of processing billions of voxels contained within a scan.

Therefore, the most of the existing FCM-based algorithms dedicated to $3 \mathrm{D}$ image segmentation are in fact $2.5 \mathrm{D}$ approaches. This means that they perform FCM segmentation slice-by-slice and then compose 3D result by combining $2 \mathrm{D}$ results obtained from single slices [6], [7]

To overcome the above mentioned limitations of FCM algorithm and make the the method available also in the case of 3D images this paper proposes a solution which incorporates the idea of super-voxels into the Fuzzy C-Means clustering approach. In particular the proposed approach extends the idea of super-pixels into supre-voxels. The Super-voxels are next clustered using FCM algorithm according to statistical features extracted using the co-occurrence matrix.

The proposed approach is next applied to extract the CSF from 3D CT datasets of brain.

The following part of this paper is divided into five sections. Firstly, in Section II, the technical background and a brief review on super-voxels and Fuzzy C-Means techniques is presented. Next, in the Section III datasets used in this paper are characterised. This is followed in Section IV by the description of the introduced approach. The results of the method are presented and discussed in Section V. Finally, Section VI concludes the paper.

\section{TheORETICAL BACKGROUND AND RELATED WORKS}

\section{A. Fuzzy C-Means Clustering}

FCM is an algorithm proposed by Bezdek [8] as an alternative for K-means clustering [9]. According to FCM algorithm, each datum point is a part of a cluster whose degree is governed by its membership grade.

What is distinct about FCM is that it divides a collection of $N$ vectors into $c$ fuzzy groups with a cluster centre for each group. It is worth noting that a datum point may be a part of many groups and it gets a membership grade ranging between 0 and 1.

The role of FCM revolves around having $c$ as the number of clusters, $c_{i}$ as the cluster centre of fuzzy group $i$ and the parameter $m$ as the weighting indicator for every fuzzy integrating group. Through optimizing the function of FCM, the fuzzy subdivision can be conducted. 
The membership $J_{F C M}(U, V)$ and the cluster centres are determined according to the following equation [9]:

$$
J_{F C M}(U, V)=\sum_{k=1}^{n} \sum_{i=1}^{c}\left(u_{i k}\right)^{m} d^{2}\left(x_{k}, v_{j}\right)
$$

where: $u_{i k}$ is a matrix of size $(c \times d), d=\left\|x_{j}, v_{i}\right\|$ is the Euclidean distance between the centroid $v_{i}$ and each pixel $x_{j}, U=u_{i k}$ represents the matrix of fuzzy partition, $V=$ $v_{1}, v_{2}, \ldots, v_{n}$ is are class centres, $m$ is the fuzzy factor with $m>1$ and $c$ is the class number.

Because of its advantages, FCM approach has been widely applied in segmentation of medical images. Application of the method to brain segmentation is especially popular. Additionally, numerous improvements to the FCM method have been proposed.

One of the major proposals in the medical image segmentation is concerning the adoption of the spatial distance into the clustering based segmentation as initiated by Tolias and Panas [10], [11], [12]. Furthermore, Liew [13] proposed an automatic segmentation of 3D dimensional Magnetic Resonance Imaging (MRI) brain images. They used a local spatial distance into the FCM algorithm adopting a new dissimilarity index instead of the standard Euclidean distance. In addition, they created a cluster prototype with variation of 3D multiplicative bias field [14].

In the same context, there is an approach which provides the extraction of some features. It can incorporate the intensity information for the voxels neighbours [15]. Moreover, the fuzzy logic supply to segment 3D image under the consideration of the following three information: position, boundary and intensity knowledge [16]. This method aims to extract the three portions of the brain such as the left cerebral hemisphere, right cerebral hemisphere, cerebellum and brain stem. One popular technique involves adopting Fuzzy C-Means stand on the local spatial continuity [14]. It takes into account the voxel neighbour information and the intensity variation.

Regarding to other methods, [17] adopted a new method for segmentation of 3D datasets, based on Fuzzy C-Means. This approach is applied only to the three views; sagital, coronal and axial. Furthermore, the extraction of CSF is reported in [6]. This approach is based on the fuzzy inference rules. It is focused on the information obtained by the fuzzy information granulation.

The use of the Fuzzy C-Means may present some constraints, especially, when applied to brain segmentation. Brain image segmentation from CT scans faces the numerous numbers of challenges due to the characteristics of the images: poor image contrast, high-level speckle noise, weakly defined boundaries and boundary gaps. The traditional Fuzzy CMeans method is often unable to perform adequately on these images complex extension. Therefore, to overcome the above drawbacks, this paper proposes a new method based on the second statistic feature by the use of the co-occurrence matrix.

\section{B. 3D Co-occurrence Matrix}

The co-occurrence matrix stores information about the occurrence of couples of pixels in the image. It takes into consideration the neighbouring pixels and the spatial relationship of pixels. In the case of intensity images, the cooccurrence matrix is also called grey-level spatial dependence matrix or grey level co-occyrence matrix (GLCM). This matrix is determined based on pixel intensity values. The idea of the GLCM determination is explained in figure 1. In particular, the $(5 \times 4)$ matrix shown on the left represents image with pixel intensities represented by numbers, while the $(6 \times 6)$ matrix on the right represents the corresponding GLCM.

\begin{tabular}{|l|l|l|l|l}
\hline 1 & 1 & 5 & 6 & 1 \\
\hline 2 & 4 & 3 & 1 & 5 \\
\hline 5 & 4 & 3 & 1 & 2 \\
\hline 6 & 3 & 1 & 2 & 4 \\
\hline 0
\end{tabular}$\quad$\begin{tabular}{lll|l|l|l|l|}
\hline 1 & 3 & 0 & 0 & 2 & 0 \\
\hline 0 & 0 & 0 & 2 & 0 & 1 \\
\hline 3 & 0 & 1 & 0 & 0 & 0 \\
\hline 0 & 0 & 2 & 0 & 0 & 0 \\
\hline 0 & 0 & 0 & 1 & 1 & 1 \\
\hline 1 & 0 & 1 & 0 & 0 & 0 \\
\hline
\end{tabular}

Gray Length Cooccurrence Matrix

Fig. 1: The idea of the gray level co-occurrence matrix determination: the input image (on the right) and the corresponding co-occurrence matrice (on the left).

The GLCM was introduced to describe two dimensional images. However, in the literature, there are approaches which concentrate on using the 3D co-occurrence matrix which involves a good description of the image information. For the first time GLCM was adopted to extract important features using the texture, called the second order statistics [18]. These include the homogeneity, the angular second moment, the entropy and the contrast. After that, the 2D Haralick texture feature was applied to medical images and extended to $3 \mathrm{D}$ domain [19]. Furthermore, it is adopted for the hyperspectral imagery as an image cube [18].

In the same context, the self organizing map (SOM) is a kind of artificial neural network founded on competing as well as unsupervised learning. The combination of SOM and FCM with the GLCM is assumed to extract the first and the second statistical features preceded by a segmentation of the input image [19]. The main inconvenient of 3D image segmentation performed in this way is that it involves only the 2D images, performing image segmentation slice-by-slice [6] [7].

\section{Super-voxels}

A super-pixel can be defined as a set of connected pixels that posses similar attributes. Most commonly, pixel intensity 
or colour is regarded [20]. Figure 2 shows the super-pixel principle. The top image represents an input $2 \mathrm{D}$ image, while the bottom image is an output image after image division into super-pixels.

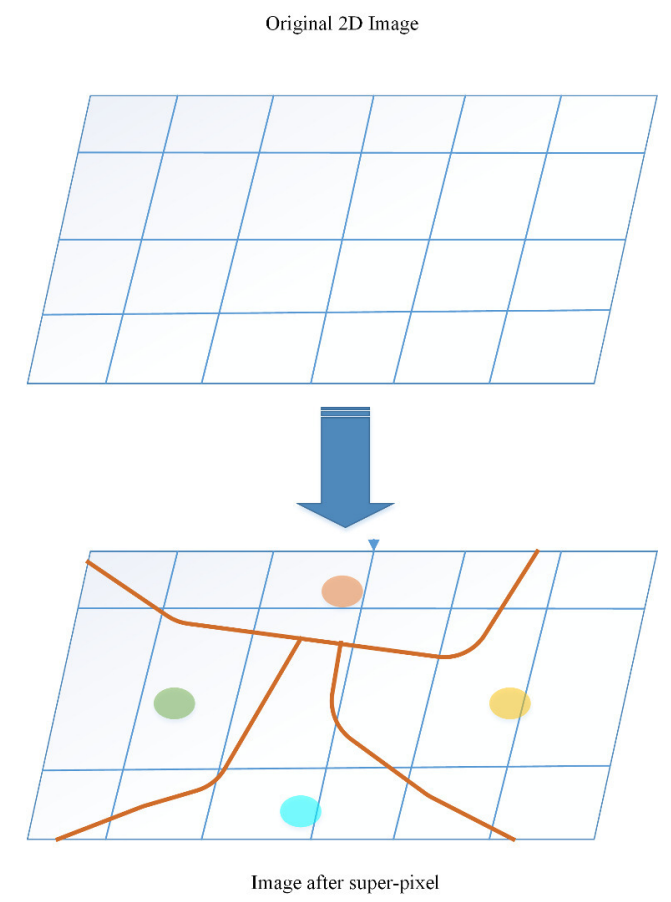

Fig. 2: A model of image segmentation using super-pixels algorithm.

The state-of-the-art is replete with approaches for image division into super-pixels, which have been proposed for $2 \mathrm{D}$ images. These include SLIC approach [21], [20] which uses linear iterative clustering, Turbo-pixels [22] which use curve evolution, NC super-pixels [23] based on the normalized graph cuts, FH super-pixels which use greedy segmentation proposed in [24] or methods based on energy minimisation framework as proposed in [25].

There are also some approaches which extend the idea of super-pixels into 3D images. For example, the approach proposed in [26] aims to divide a three dimensional image into blocks. Other method uses the super-voxel technique for processing a Voxel Of Interest (VOI) [27] instead of the whole voxels of the image. In [28], the convexity is considered as a metric for the super-voxel extraction. Moreover, a method of super-voxel combined with a clustering approach has been reported in [29] in order to extract statistical features. The same problem is treated in [30]. It aims to determine the region of interest adopting shift followed by the super-pixel algorithm. Its advantage is that it is applicable not only for 3 dimensions, but also from 1 to $N$ dimensions.

The method proposed in this paper extends Zero version of Simple Linear Iterative Clustering (SLICO) approach into three dimensions. SLICO method is widely used in the literature. It aims to divide the input image into a super-pixels, that commonly have a uniform and compact shape with better boundary stickiness. In this paper, we adopted the SLICO technique because it is fast to compute, memory efficient, and simple to use. The memory efficiency and low computational cost is especially important when segmentation of 3D images is considered.

Figure 3 shows the application of SLICO approach to a sample CT brain slice. In particular, the figure (3-a) represents the input $2 \mathrm{D}$ image, while the figure (3-b) shows the result of SLICO method.

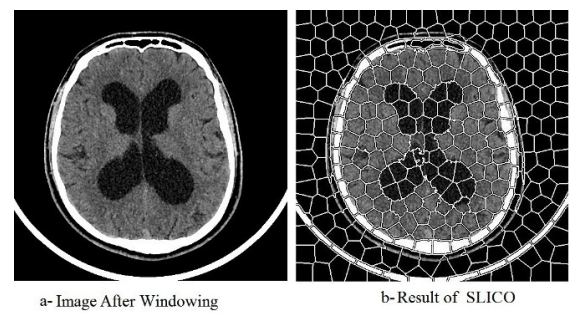

Fig. 3: SLICO approach for 2D image super-pixel segmentation: a- Image after windowing, b- Result of SLICO algorithm.

To the best of our knowledge the combination between the FCM and super-pixel is concerned only in [31] and [32]. The first work takes into account the super-pixel technique as a clustering objects in spite of the classical super-pixel. The second approach inspects a different strategy. An additional feature of segmentation is added (eg. extraction of CSF, white matter and gray matter). Both methods however are dedicated only to segmentation of 2D brain images.

\section{INPUT DATA}

Ten CT brain scans in Digital Imaging and Communications in Medicine (DICOM ) format were used in this paper. All of them present brain with the ventricular system enlarged due to the hydrocephalus. The images were adopted in order to extract the CSF contained within the brain ventricles, to test the proposed approach and to evaluate its performance in comparison to other methods. The average number of slice in the dataset was 215. Each slice had the spatial resolution of $512 \times 512$, the bit resolution of 12 and the slice thickness equal to 1.5000 . In addition, the spacing between slices has $0.7500 \mathrm{~mm}$.

The figure 4 shows $2 \mathrm{D}$ the selected slices composing a sample 3D CT brain scan. The slices are after intensity windowing.

\section{Proposed Method}

The Modified Fuzzy C-Means algorithm based on supervoxels is our proposed approach. The main idea behind this approach is to perform image division into super-voxels using the extended SLICO approach and then cluster the resulting regions using FCM algorithm.

The proposed method contains three main steps, namely: image pre-processing followed by an application of the supervoxels technique and finished by using the modified Fuzzy C-Means algorithm. 

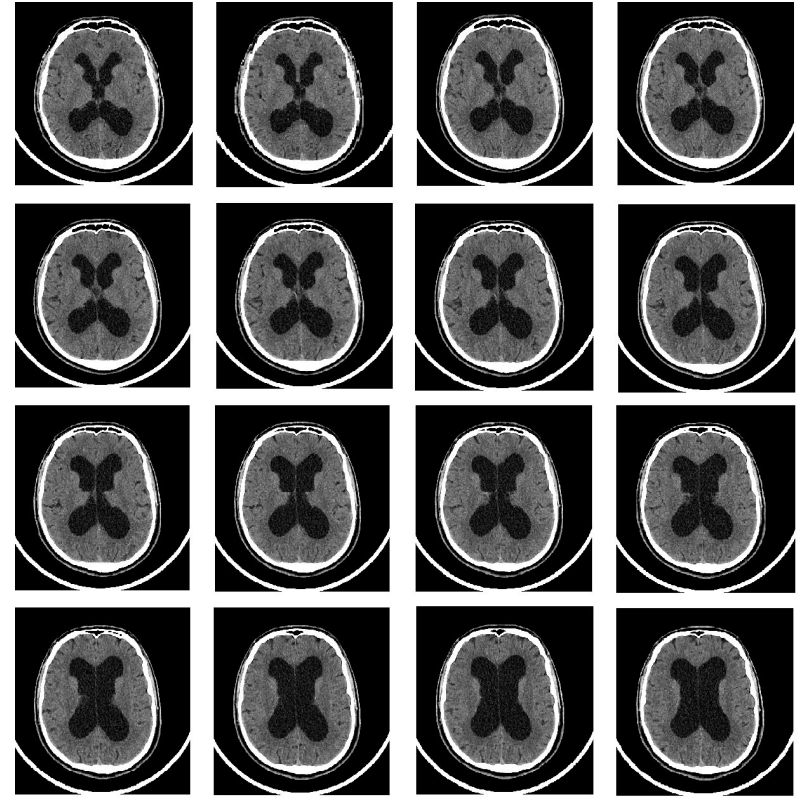

Fig. 4: Sample CT brain slices after pre-processing step.

The block diagram of the introduced approach is shown in figure 5. The details regarding each step of the introduced approach are given in the following subsections.

\section{A. Image pre-processing}

The pre-processing is the fundamental task for the introduced approach. It is mainly operated by window and contrast adjustment. The modification of the window as well as the contrast values depends on the input image and the region of interest. The information about the desired window is usually given in the DICOM header.

In the preprocessing step, firstly image intensities are linearly transformed according to the rescale intercept and slope as described in equation ( 2 ).

$$
N e w_{H U}=\left(R P V \times R_{S}\right)+R_{I}
$$

where: RPV is the raw (original) pixel value, $R_{S}$ is the rescale slope and $R_{I}$ represents the rescale intercept.

After applying the rescale/intercept transformation, image windowing is performed. Generally, crucial brain regions such as the cerebrospinal fluid, the white matter and the grey matter drop within the interval from 0 to 150 under Hounsfields Units (HU). Accordingly, the windowing procedure has to be achieved to highlight intensities within the region of interest. In particular, the original pixel values declined over the range are threshold to black or white. To obtain this, the window centre $W_{C}$ is set to 40 , while, the window level $W_{L}$ is set to 80. Finally, the images converted to 8-bit grey scale format, where intensities range from 0 (black) to 255 (white). This procedure is described by equation ( 3 ).

$$
\text { GrayImage }=255 \frac{W_{M a x}-W_{\text {Min }}}{N e w_{H U}-W_{\text {Min }}}
$$

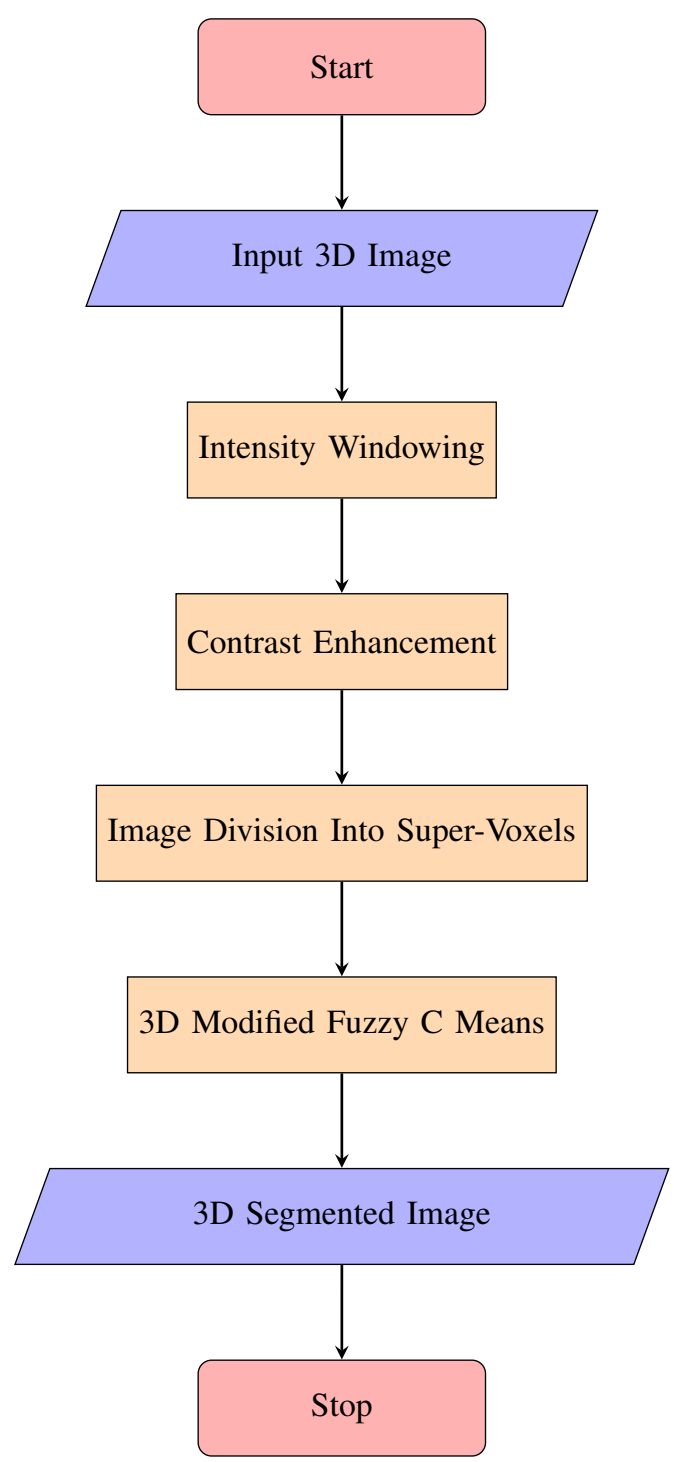

Fig. 5: The block diagram of the proposed method.

where: $W_{M a x}=W_{C}+W_{L} / 2$ and $W_{M i n}=W_{C}-W_{L} / 2$.

Figure 6 illustrates the original image and the image after the windowing. In particular, sub-figures 6 a and $6 \mathrm{c}$ show sample images before windowing, while sub-figures $6 \mathrm{~b}$ and 6 $\mathrm{d}$ correspond to images after this procedure.

After intensity transformation, intensities corresponding with brain region and CSF region are highlighted.

\section{B. Super-voxels algorithm}

In this paper, the SLICO super-pixels algorithm is extended to be adequate with three dimensional images and greyscale super-voxels. In order to do this, the initialization of the cluster is required. Thus, we called SLICO technique a super-pixel clustering. The second step is to calculate the spatial distance between the cluster centre and each voxel in the window of size $(7 \times 7 \times 7)$. Eventually, the new cluster centres have to be updated relatively to the spatial distance. 


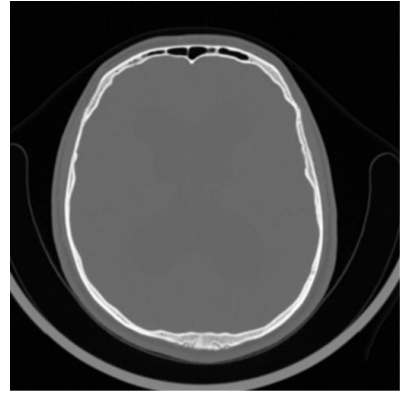

a- Original Image
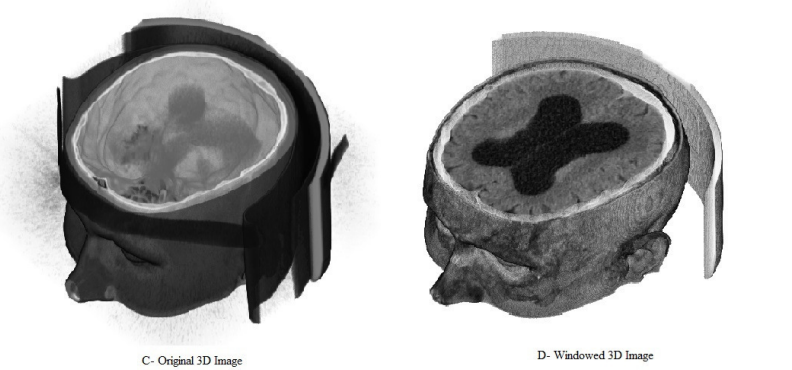

Fig. 6: Image windowing: a- 2D original image, b- 2D windowed image, c- 3D original image, d- 3D windowed image.

The proposed method presents several advantages compared to existing ones. Simple linear iterative clustering (SLICO) is an adaptation of k-means for super-voxels generation, with two important distinctions:

- The number of distance calculations in the optimization is greatly decreased. This reduce is due to the limiting the search space to a region proportional to the super-voxels size. This minimizes the complexity to be linear in the number of pixels $N$ and also independent of the number of super-voxels $k$.

- While simultaneously a providing control over the size and compactness of the super-voxels, a weighted distance measure combines colour and spatial proximity. SLICO is similar to the approach described in [17]. This latter is used as a pre-processing step for depth estimation, which was not fully explored in the context of supervoxel generation.

The algorithm 1 can be described step by step as follows [33]:

1) Use the vector $[l x, a x, b x, c x, x k, y k, z k]$ to represent each voxel, $[l x, a x, b x, c x]$ for the voxel colour vector (in the case of greyscale image, this vector $=[0,0,1])$, $[x k, y k, z k]$ is the voxel position, then the voxel of our colour similarity and distance to produce super-voxels. The grid interval is $(S=\sqrt[N / K]{2})$; initialize the $K$ clusters centres.

2) In the area of where $(n * n * n)$, find the minimum gradient position $I(x k, y k, z k)$ is $(x k, y k, z k)$ position of the voxel $[l x, a x, b x]$ vector, $\|$.$\| is the norm.$

3) Perform the following steps to know the cycle $E$
Algorithm 1: Super-Voxels Algorithm (SLICO)

\section{procedure}

$/ *$ Initialisation*/

Initialize cluster coordinates $C_{i}=[l x, a x, b x, c x, x k, y k, z k]^{T}$

Sampling voxels at regular grid steps $S$

Move cluster centres to the lowest gradient position in a $(7 * 7 * 7)$ neighbourhood

for each voxel $i$ do

label $l(i)=-1$

Set distance $d(i)=$ inf

end for

repeat

/*Assignment*/

for each cluster center $C_{k}$ do

for each voxel in a $(7 S * 7 S * 7 S)$ region around $C_{k}$ do Compute the distance $D$ between $C_{k}$ and $i$

$$
\begin{gathered}
\text { if } D<d(i) \text { then } \\
\text { set } d(i)=D \\
\text { set } l(i)=k
\end{gathered}
$$

end if

end for

end for

/*Update*/

Compute new cluster centers

Compute residual error $E$

D1 distance between previous centers

recomputed centers

Until $E \leftarrow$ threshold

Enforce Connectivity

end procedure

(residual error) $<a$ threshold:

Each cluster centre $C_{k}$ is designed for $(7 S \times 7 S \times 7 S)$ voxels area. The most appropriate voxel allocated to this cluster is for the greater value of $m$.

4) After the clustering is complete, recalculate the cluster centres and $E$;

5) Connect similar regions.

The adopted technique is described by Algorithm 1.

The improved SLICO technique adopts the typical compactness parameters (chosen as an initialization) applied to all super-voxels in the 3D image. In the case of a high smoothness in some regions with a high texture for the others, the SLICO provides a smoothness repeatedly super-voxels in the weak regions and extremely intermittent super-voxels in the textured regions.

SLICO is an improvement of SLIC proposed in [33] to solve that problem effectively. The compactness parameter must not be initialized by the user. SLICO precisely selects the compactness parameter adequate for each super-voxel separately. This achieves ordinary shaped super-voxels for both 
textured and non textured parts in the image.

The figure 7 represents the application of SLICO algorithm after its extension to three dimensions as proposed in this paper. The figures 7-a and 7-c show the original 2D and 3D images after windowing, while, the figures 7-b and 7-d show the $3 \mathrm{D}$ image after division into SLICO super-voxels.

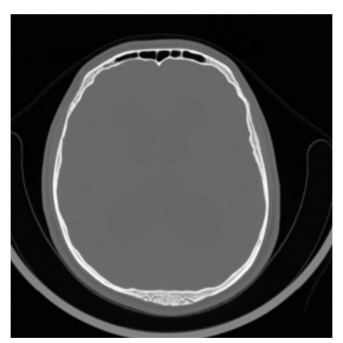

a- Input 2D Image

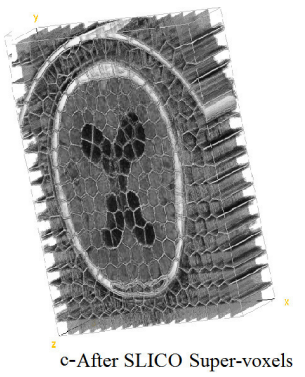

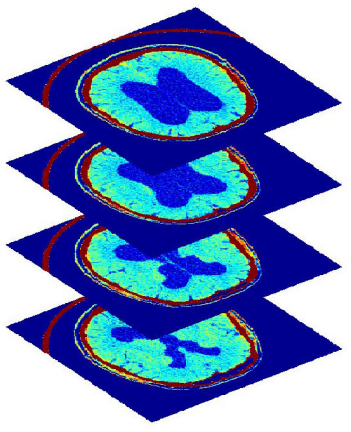

b-3D Image After Windowing

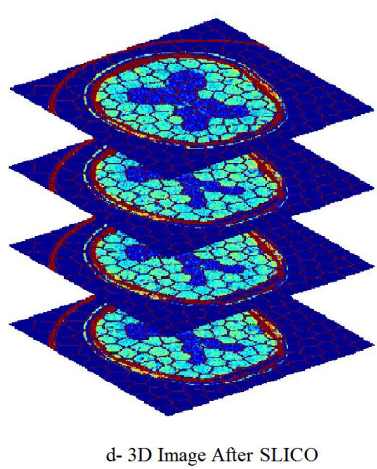

Fig. 7: Results of the extended SLICO algorithm applied to 3D Image: a- input 2D image, b-after SLICO super-voxel, c-3D image after windowing, d-3D image after SLICO.

\section{Modified Fuzzy C-Means}

The modified Fuzzy C-Means is a combination between a creation of the co-occurrence matrix and the standard Fuzzy C-Means algorithm.

Conventionally, many approaches of 2D image segmentation take into account the two specified parts: the segmentation technique and the representation system. Throughout this structure, the proposed approach is defined as an amendment of the Fuzzy C-Means algorithm, established on a cooccurrence matrix [34]. The Fuzzy C-Means algorithm can be adopted to compute the membership degree for each supervoxel. However, the FCM algorithm involved only the grey level and does not include the super-voxels spatial information with consideration of each other. For this reason, we determined the statistical attributes of the image after applying the super-voxels technique. This combination may help to impress this inconvenient.

The steps of 3D Modified Fuzzy C-Means method are specified as follows [34]:
1) Choose our input image after super-voxel technique.

2) Set the size of the sliding window.

3) Calculate of the co-occurrence matrix for the sake of extracting a peculiar image.

4) Perform the standard Fuzzy C-Means algorithm which is applied to the attribute image to attain the final segmented one.

5) Adopt the standard Fuzzy C-Means technique in order to extract the region of interest (CSF).

1) Spatial feature correlation method: In this paper, we used the co-occurrence matrix [35] as it is related to the presence of a voxel pair from the given image $I$. The cooccurrence matrix is made of important data that restore the class bias of $I$. Consequently, the proposed co-occurrence matrix performs a major role in image dividing.

The co-occurrence matrix, known also as spatial feature correlation method, describes the occurrence of voxel pairs in the distance denoted $d$ in a certain direction in accordance to the following equation:

$\operatorname{Cooc}(i, j, k, R)=\operatorname{card}\left\{\begin{array}{r}\left((x, y, z),\left(x^{\prime}, y^{\prime}, z^{\prime}\right) \in D, \text { checking } R(d, \theta)\right. \\ I(x, y, z)=i ; I\left(x^{\prime}, y^{\prime}, z^{\prime}\right)=j\end{array}\right.$

where $\operatorname{card}(A)$ denotes the cardinality of the subset $A$, checking $R(d, \theta)$ expresses the relation between two voxels, $d$ is the Euclidean distance between two voxels. $\theta$ is the angle that describes the orientation of the two voxels among the horizontal direction. This angle can be equal to $0^{\circ}$ or $45^{\circ}$ or $90^{\circ}$ or $135^{\circ}$. Every element of the co-occurrence matrix $\operatorname{Cooc}(i, j, k, R)$ conforms to the number of voxel pairs $(i, j, k)$. It expresses the number of occurrence of a voxel which has a gray-level value $j$. Therefore, this occurrence have to be related to a horizontal adjacency. Subsequently, the evaluation of the regions within the image is made through the use of the co-occurrence matrix. Therefore, the removal of the second statistical features will be simple. These features are the mean $M e$ (Eqn. 5), the variance $V$ (Eqn. 6), the Skinewski Sk (Eqn. 7) and the Kurtosis $K u$ (Eqn. 8).

$$
\begin{gathered}
M e=\frac{1}{M \times N \times S} \sum_{k=1}^{s} \sum_{i=-\frac{w-1}{2}}^{\frac{w-1}{2}} \sum_{j=-\frac{w-1}{2}}^{\frac{w-1}{2}} I(n+i, r+j, t+k) \\
V=\frac{1}{M \times N \times S} \sum_{k=1}^{s} \sum_{i=-\frac{w-1}{2}}^{\frac{w-1}{2}} \sum_{j=-\frac{w-1}{2}}^{\frac{w-1}{2}}(I(n+i, r+j, t+k)-M e)^{2}
\end{gathered}
$$

$S k=\frac{1}{M \times N \times S} \sum_{k=1}^{s} \sum_{i=-\frac{w-1}{2}}^{\frac{w-1}{2}} \sum_{j=-\frac{w-1}{2}}^{\frac{w-1}{2}}(I(n+i, r+j, t+k)-M e)^{3}$ 
$K u=\frac{1}{M \times N \times S} \sum_{k=1}^{s} \sum_{i=-\frac{w-1}{2}}^{\frac{w-1}{2}} \sum_{j=-\frac{w-1}{2}}^{\frac{w-1}{2}}(I(n+i, r+j, t+k)-M e)^{4}$

where $I$ denotes the image and $(M \times N \times S)$ represents the size of $I,(w \times w \times w)$ is the sliding windows as shown in the figure 9 . The four features are obtained from the windows size $(7 \times 7 \times 7)$, described by the figure 8 .

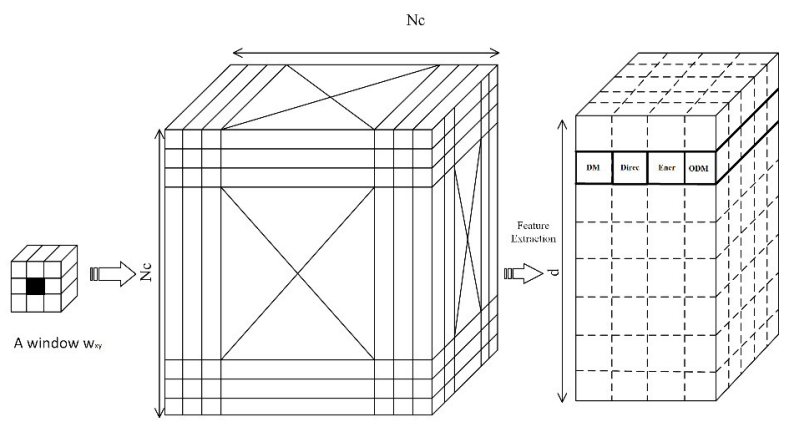

The co-occurrence matrix

Fig. 8: A sliding window for statistical features extraction.

The window is centered at the voxels $(n, r, t)$ in order to extract a centred window around every voxels. Hence, in the figure 8 , the vector that contains the statistical features (DM, Direc, Ener, ODM) is classified adopting the C-Means algorithm into $c$ classes.

The segmentation based on the C-Means algorithm divides the image in $c$ regions (classes). The dimensional scanning plan of an image is implemented voxel by voxel.

2) Standard Fuzzy C-Means Algorithm: After applying the SLICO super-voxels algorithm for dividing the image into super-voxels, we extracted the attribute image (Means, Variance, Skinewski and Kurtosis). The third step of the proposed method is to adopt the Fuzzy C-Means algorithm to each super-voxels of the obtained image.

The Fuzzy C-Means aims to minimize the weighted within class sum of squared error objective function [3] :

$$
J_{F C M}(U, V)=\sum_{l=1}^{s} \sum_{k=1}^{n} \sum_{i=1}^{c}\left(u_{l i k}\right)^{m}\left\|x_{k}-v_{i}\right\|^{2}
$$

where $x=\left[x_{1}, x_{2}, x_{3}, \ldots, x_{n}\right]^{T}$ is the data set, $U$ is composed by memberships $u_{i l k}$ of $k^{\text {th }}$ bit in the $i^{\text {th }}$ class and $m$ is the fuzzy factor with $m>1$.

The proposed solution of the objective function can be attained using an iterative process, that is performed as follows:

1) Input of the original image which has a size $(M \times N \times$ $D)$,

2) Initialize the parameters: the fuzzifier and the centres of classes,

3) Initialize the partition matrix $U^{(0)}$ based on random variables between 0 and 1 ,
4) Calculate of the Euclidean distance referring to the following equation :

$$
d(x, y, z)=\sqrt{\left(z_{2}-z_{1}\right)^{2}+\left(y_{2}-y_{1}\right)^{2}+\left(x_{2}-x_{1}\right)^{2}},
$$

where: $\left(x_{1}, y_{1}, z_{1}\right)$ are the coordinates of the first voxel, while $\left(x_{2}, y_{2}, z_{2}\right)$ are the coordinates of the second voxel.

5) Update of the prototype using the equation as follows:

$$
b_{i}=\frac{\sum_{k=1}^{n} U_{i k}^{m} \times x_{k}}{\sum_{k=1}^{n} U_{i k}^{m}}
$$

6) Calculate the partition matrix $U^{(k)}$ according to equation:

$$
U_{l i j}=\left[\sum_{l=1}^{s} \sum_{k=1}^{c}\left(\frac{d^{2}\left(x_{j}, b_{i}, z_{l}\right)}{d^{2}\left(x_{j}, b_{k}, z_{l}\right)}\right)^{\frac{2}{(m-1)}}\right]^{-1}
$$

7) Convergence test: repetition of the 4,5 and 6 steps described by the following equation:

$$
\left\|U^{(k+1)}-U^{(k)}\right\|<\varepsilon
$$

where $\varepsilon$ is the tolerance. It converges to zero.

\section{EXPERIMENTAL RESULTS}

This section presents the results of applying the introduced approach to 10 sample CT images of brain. In particular, a region of CSF is extracted by the proposed method.The sub-figure (10-a) is the windowed 3D image, the sub-figure (10-b) is the result after applying the SLICO algorithm. The sub-figure (10-c) shows the image after applying the SLICO algorithm combined with the mathematical morphology (10d) is the image after Modified Fuzzy C-Means algorithm. The Figure 11 presents an the results shown in 3D. While, the figure 12 represents the sample slice overlayed.

The proposed approaches were compared in terms of the accuracy and the execution time with the following approaches: Modified Fuzzy-C Means, the combination between SLIC and MFCM and the combination between SLICO and MFCM.

The results of accuracy comparison (in percentage) are given in the Table I. It was measured as follows :

$$
\text { Accuracy }=\frac{\text { Number of correctley classified pixels }}{\text { Total number of pixels }} \times 100 \%
$$

The first column shows the case ID. This is followed by the accuracy of the MFCM. The third column represents the accuracy of SLIC combined with the MFCM and the last column shows the accuracy percentage of our proposed method (SLICO+MFCM). While Table II presents comparison of execution time between the Modified Fuzzy C Means, the combination between the Modified Fuzzy C-Means, the Modified Fuzzy C Means combined with the SLIC supervoxels algorithm and the the combination between SLICO and MFCM.

The execution time is given in the table II. Tests were 


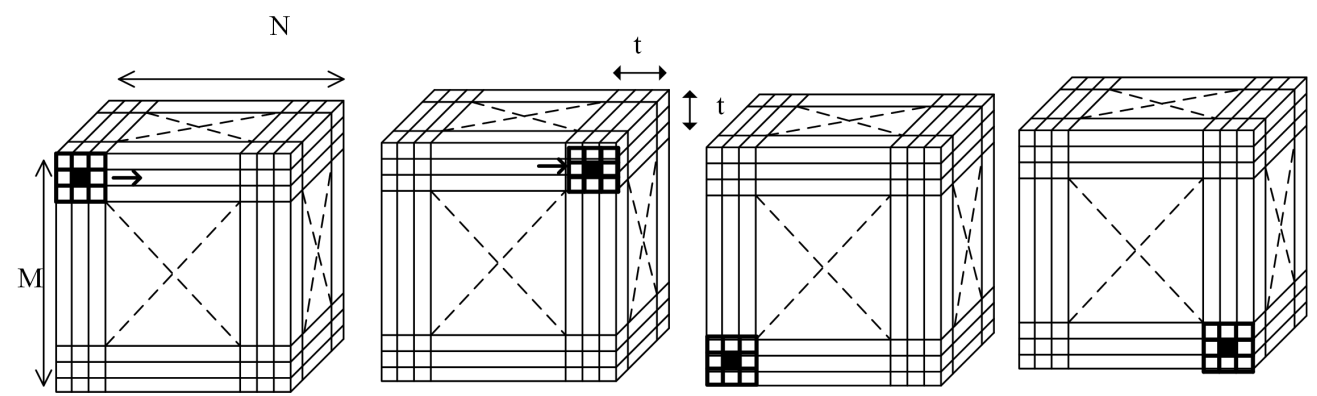

Fig. 9: The adaptive sliding windows from the left to the right and from the top to the bottom on an $\left(M^{*} N^{*} D\right)$ Image.

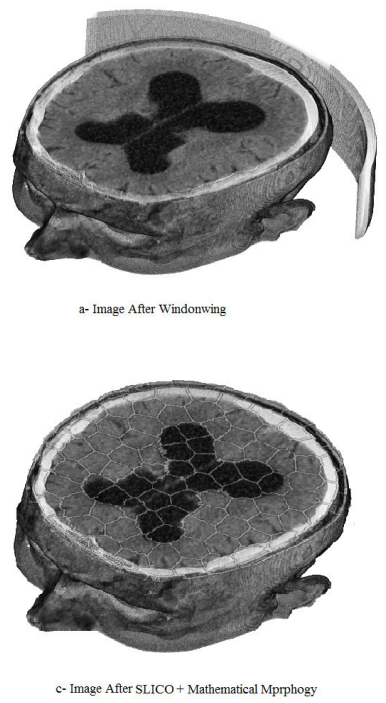

Fig. 10: Image segmentation result: a- Image after windowing, b- Image after SLICO supervoxels, c- After SLICO + mathematical morphology, d- After Modified Fuzzy C-Means.
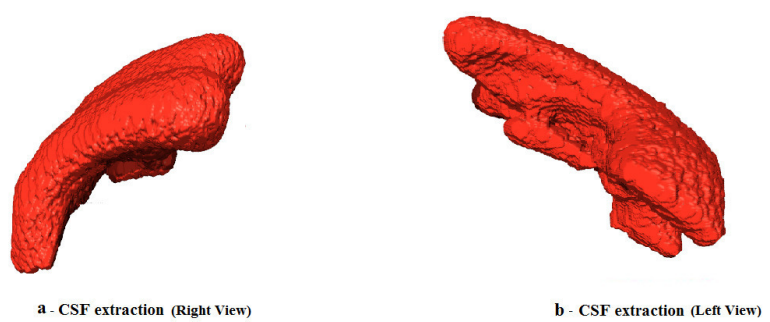

Fig. 11: SLICO combined with the MFCM Results in 3D.

performed on a PC computer with an Intel Core (TM) i5$3450 \mathrm{CPU} 3.10 \mathrm{GHz}$, a $32 \mathrm{~GB}$ of RAM and a CUDA for Graphic Processing Unit using Graphic Parallel Unit Toolbox under Matlab 2013a version.

We can interpret the figure 12 and 13 that the two classes are correctly extracted for $2 \mathrm{D}$ and $3 \mathrm{D}$ images. The first class is the CSF region and the second one if for the rest of the image.

In our paper, we are interested in the CSF region. So, the
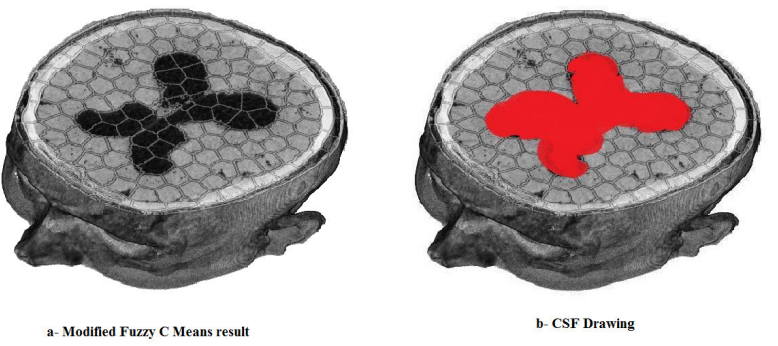

Fig. 12: Sample slice overlayed.

figure 13 takes into account the region of Interest (CSF). It is clear that, our SLICO technique combined with the Modified Fuzzy C Means is more efficient than the SLIC technique combined with the Modified Fuzzy C-Means.

From the Table I, we can say that the 3D Modified Fuzzy $\mathrm{C}$ Means takes much time than the ameliorated version based on the GPU. Otherwise, the MFCM combined with SLICO technique is faster than the SLIC technique combined with the MFCM algorithm. The average time of the combination between SLICO technique and Modified Fuzzy C-Means is about 20.94 s. Althought, for The average time of the combination between SLIC technique and Modified Fuzzy C-Means is about 29.10 .

Furthermore, the Table II demonstrates that the ameliorated MFCM combined with the SLICO is more accurate than the combination between the MFCM algorithm and SLIC supervoxels technique.

The extracted CSF from three dimensional image is showed in the figure 13. As can be seen in this figure, the visualization of the (VOI)Volume Of Interest using our prposed method (MFCM+SLICO) is more consistent than the Modified Fuzzy C-Means combined with the SLIC technique.

\section{CONCLUSION}

The segmentation method proposed in this article, is a novel region segmentation method based on the super-voxel technique and the modified Fuzzy C-Means algorithm while the Cerebro-Spinal Fluid (CSF) part has a good consistency. This method consists of three steps. In the first step, the intensity windowing and contrast enhancement are applied 

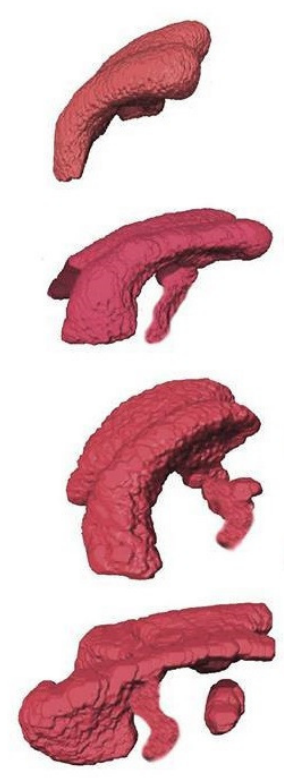

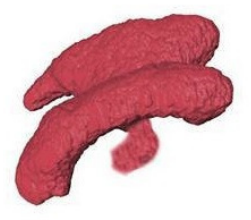

a)
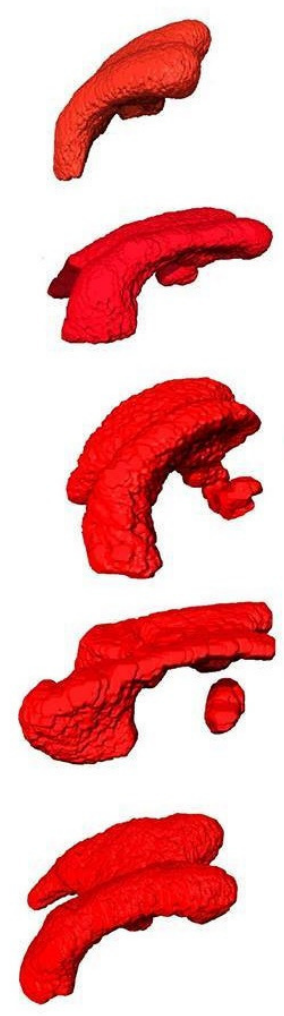

b)
Fig. 13: CSF Visualization: a) SLIC combined with MFCM, b) SLICO combined with MFCM results.

TABLE I: Comparison the accuracy between original MFCM, SLIC algorithm combined with MFCM and SLICO algorithm combined with MFCM.

\begin{tabular}{|c|c|c|c|}
\hline Case ID & $\begin{array}{c}\text { Accuracy } \\
\text { MFCM } \\
(\%)\end{array}$ & $\begin{array}{c}\text { Accuracy } \\
\text { SLIC+MFCM } \\
(\%)\end{array}$ & $\begin{array}{c}\text { Accuracy } \\
\text { SLICO+MFCM } \\
(\%)\end{array}$ \\
\hline 01 & 93,12 & 96,13 & 97,50 \\
\hline 02 & 92,60 & 93,15 & 98,27 \\
\hline 03 & 88,54 & 90,04 & 96,87 \\
\hline 04 & 90,45 & 95,68 & 97,31 \\
\hline 05 & 75,21 & 80,05 & 82,86 \\
\hline 06 & 80,05 & 82,78 & 89,17 \\
\hline 07 & 91,80 & 93,43 & 95,01 \\
\hline 08 & 82,34 & 86,15 & 91,33 \\
\hline 09 & 76,23 & 89,90 & 90,17 \\
\hline 10 & 78,14 & 81,94 & 85,35 \\
\hline
\end{tabular}

TABLE II: Comparison the execution time between original MFCM, SLIC algorithm combined with MFCM and SLICO algorithm combined with MFCM.

\begin{tabular}{|c|c|c|c|}
\hline Case ID & $\begin{array}{c}\text { Time } \\
\text { MFCM } \\
(\mathrm{s})\end{array}$ & $\begin{array}{c}\text { Time } \\
\text { SLICO+MFCM } \\
(\mathrm{s})\end{array}$ & $\begin{array}{c}\text { Time } \\
\text { SLIC+MFCM } \\
(\mathrm{s})\end{array}$ \\
\hline 01 & 1120,56 & 11,13 & 11,50 \\
\hline 02 & 1240,60 & 14,30 & 14,55 \\
\hline 03 & 1224,34 & 12,84 & 13,40 \\
\hline 04 & 1149,57 & 11,68 & 12,16 \\
\hline 05 & 1180,68 & 10,05 & 10,76 \\
\hline 06 & 1202,85 & 12,78 & 13,23 \\
\hline 07 & 1136,90 & 13,43 & 15,02 \\
\hline 08 & 1210,42 & 13,15 & 13,44 \\
\hline 09 & 1119,35 & 11,90 & 12,27 \\
\hline 10 & 1127,14 & 11,94 & 12,68 \\
\hline
\end{tabular}

to the input 3D CT image. In the second step, we adopted an image division into super-voxels. Then, a segmentation modified Fuzzy C-Means approach is applied in order to segment the image into two classes. Considerable evaluation results have demonstrated great potential on our new approach.

Regarding to the main objective of this research paper, there is no exist method suggested the combination of fuzzy logic rules with a super-voxel technique. Furthermore, the proposed method considers the neighbouring membership degree among the voxels of the images during the determination of a final classification which can be unable with traditional segmentation methods.

\section{ACKNOWLEDGMENT}

This work is financially funded by the European Union under the Erasmus Mundus project. This research was funded also by the Ministry of Science and Higher Education of Poland from founds for science in years 2013-2015 in a framework of Iuventus Plus programme (project no. IP 2012 011272).

\section{REFERENCES}

[1] S. M. S. Szilágyi, László and Z. Benyó, "A modified fuzzy c-means algorithm for mr brain image segmentation," in Image Analysis and Recognition, vol. 4633, August 2007, pp. 866-877.

[2] S. C. L. Lee, Lay Khoon and W. J. Thong, "A review of image segmentation methodologies in medical image." Advanced Computer and Communication Engineering Technology.

[3] B. Irving, A. Cifor, B. W. Papież, J. Franklin, E. M. Anderson, M. Brady, and J. A. Schnabel, "Automated colorectal tumour segmentation in dcemri using supervoxel neighbourhood contrast characteristics," in Medical Image Computing and Computer-Assisted Intervention MICCAI 2014. Springer International Publishing, 2014, pp. 609-616.

[4] P. B. Kanade and P. P. Gumaste., "Brain tumor detection using mri images." International Journal of Innovative Research in Electrical, Electronics, Instrumentation and Control Engineering, vol. 03, pp. 146150, 022015.

[5] S. K. Adhikari, J. K. Sing, D. K. Basu, and M. Nasipuri, "A spatial fuzzy c-means algorithm with application to mri image segmentation," in Advances in Pattern Recognition (ICAPR), 2015 Eighth International Conference on. IEEE, 2015.

[6] Y. Hata, S. Kobashi, S. Hirano, H. Kitagaki, and E. Mori, "Automated segmentation of human brain $\mathrm{mr}$ images aided by fuzzy information granulation and fuzzy inference." Systems, Man, and Cybernetics, Part C: Applications and Reviews, IEEE Transactions on, vol. 30, no. 02, pp. 381-395, 022000.

[7] S. Kobashi, Y. Fujiki, M. Matsui, N. Inoue, K. Kondo, Y. Hata, and T. Sawada, "Interactive segmentation of the cerebral lobes with fuzzy inference in 3t mr images." Systems, Man, and Cybernetics, Part B: Cybernetics, IEEE Transactions on, vol. 36, no. 01, pp. 74-86, 022006. 
[8] J. C. Bezdek, "Pattern recognition with fuzzy objective function algorithms," New York: Plenum Press, 1981.

[9] J. C. Dunn, "A fuzzy relative of the isodata process and its use in detecting compact well-separated clusters," Journal of Cybernetics, pp. 32-57, September 1974.

[10] S. M. P. Yannis A Tolias, "On applying spatial constraints in fuzzy image clustering using a fuzzy rule-based system," Signal Processing Letters, IEEE, vol. 5, pp. 245-247, October 1998.

[11] A. Abdullah, A. Hirayama, S. Yatsushiro, M. Matsumae, and K. Kuroda, "Cerebrospinal fluid pulsatile segmentation-a review," in Biomedical Engineering International Conference (BMEiCON), 2012. IEEE, 2012, pp. 1-7.

[12] H. A. Y. S. M. M. Abdullah, A. and K. Kuroda, "Cerebrospinal fluid image segmentation using spatial fuzzy clustering method with improved evolutionary expectation maximization," in Engineering in Medicine and Biology Society (EMBC) 2013 35th Annual International Conference of the IEEE. IEEE, 2013, pp. 3359-3362.

[13] S. H. L. Liew, AW-C. and W. H. Lau, "Segmentation of color lip images by spatial fuzzy clustering." Fuzzy Systems, IEEE Transactions on, vol. 11, pp. 542-549, 2003.

[14] A. W.-C. Liew, S. H. Leung, and W. H. Lau, "Fuzzy image clustering incorporating spatial continuity," IEE Proceedings-Vision, Image and Signal Processing, vol. 147, no. 2, pp. 185-192, 2000

[15] P. Shen and C. Li, "Local feature extraction and information bottleneckbased segmentation of brain magnetic resonance (mr) images," Entropy, vol. 15 , no. 8, pp. 3205-3218, 2013.

[16] G. Aubert and P. Kornprobst, "Mathematical problems in image processing: partial differential equations and the calculus of variations," Springer-Verlag New York Inc., 2006.

[17] H. Khotanlou, O. Colliot, J. Atif, and I. Bloch, "3d brain tumor segmentation in mri using fuzzy classification, symmetry analysis an spatially constrained deformable models," Fuzzy Sets and Systems, vol. 160, no. 10, pp. 1457-1473, 2009.

[18] F. Tsai, C.-K. Chang, J.-Y. Rau, T.-H. Lin, and G.-R. Liu, "3d computation of gray level co-occurrence in hyperspectral image cubes," in Energy Minimization Methods in Computer Vision and Pattern Recognition. Springer, 2007, pp. 429-440.

[19] L. Tesař, A. Shimizu, D. Smutek, H. Kobatake, and S. Nawano, "Medical image analysis of $3 \mathrm{~d}$ ct images based on extension of haralick texture features," Computerized Medical Imaging and Graphics, vol. 32, no. 6 , pp. 513-520, 2008

[20] R. Achanta, A. Shaji, K. Smith, A. Lucchi, P. Fua, and S. Süsstrunk, "Slic superpixels," 2010

[21] C. Y. Ren and I. Reid, "Slic: a real-time implementation of slic superpixel segmentation," University of Oxford, Department of Engineering, Technical Report, 2011.

[22] A. Levinshtein, A. Stere, K. Kutulakos, D. Fleet, S. Dickinson, and K. Siddiqi, "Turbopixels: Fast superpixels using geometric flows." IEEE
Transactions on Pattern Analysis. and Machine Intelligence, vol. 31(12), pp. 2290-2297, 2009

[23] X. Ren and J. Malik, "Learning a classification model for segmentation." in Proceedings of the 9th IEEE International Conference on Computer Vision, 2003, pp. 10-17.

[24] P. F. Felzenszwalb and D. P. Huttenlocher, "Efficient graph-based image segmentation." International Journal of Computer Vision, vol. 59(2), pp. 167-181, 2004.

[25] O. Veksler, Y. Boykov, Mehrani., and P., "Supervoxels in an energy optimization framework." in Proceedings of the 11th European Conference on Computer Vision, 2010, pp. 211-224.

[26] A. Fabijanska and J. Goclawski, "The segmentation of 3d images using the random walking technique on a randomly created image adjacency graph," 2014

[27] D. Mahapatra, P. J. Schuffler, J. A. Tielbeek, J. C. Makanyanga, J. Stoker, S. A. Taylor, F. M. Vos, and J. M. Buhmann, "Automatic detection and segmentation of crohn's disease tissues from abdominal mri," Medical Imaging, IEEE Transactions on, vol. 32, no. 12, pp. 2332-2347, 2013.

[28] H. E. Tasli, C. Cigla, and A. A. Alatan, "Convexity constrained efficient superpixel and supervoxel extraction," Signal Processing: Image Communication, vol. 33, pp. 71-85, 2015.

[29] B. Andres, U. Koethe, T. Kroeger, M. Helmstaedter, K. L. Briggman, W. Denk, and F. A. Hamprecht, "3d segmentation of sbfsem images of neuropil by a graphical model over supervoxel boundaries," Medical image analysis, vol. 16, no. 4, pp. 796-805, 2012.

[30] A. Foncubierta-Rodríguez, H. Müller, and A. Depeursinge, "Regionbased volumetric medical image retrieval," in SPIE Medical Imaging. International Society for Optics and Photonics, 2013, pp. 867406 867406.

[31] S. Jia and C. Zhang, "Fast and robust image segmentation using an superpixel based fcm algorithm," in Image Processing (ICIP), 2014 IEEE International Conference on. IEEE, 2014, pp. 947-951.

[32] S. Ji, B. Wei, Z. Yu, G. Yang, and Y. Yin, "A new multistage medical segmentation method based on superpixel and fuzzy clustering," Computational and mathematical methods in medicine, vol. 2014, 2014.

[33] R. Achanta, A. Shaji, K. Smith, A. Lucchi, P. Fua, and S. Susstrunk, "Slic superpixels compared to state-of-the-art superpixel methods," Pattern Analysis and Machine Intelligence, IEEE Transactions on, vol. 34 no. 11, pp. 2274-2282, 2012.

[34] A. Bakkari, E. Ben Braiek, I. Njeh, and A. Ben Hamida, "Automatic brain $\mathrm{mr}$ perfusion image segmentation using adaptive diffusion flow active contours based on modified fuzzy c means," in Advanced Technologies for Signal and Image Processing (ATSIP), 2014 1st International Conference on. IEEE, 2014, pp. 214-218.

[35] M. M. Morales DI and P. F, "Urban and non urban area classification by texture characterishics and data fusion," International Geoscience and Remote Sensing Symposium, vol. 6, pp. 3504-3506, 2003. 